\title{
The impact of an N1 lymph node examination in patients with early-stage non-small cell lung cancer: a retrospective cohort study
}

\author{
Wenyu Zhai ${ }^{1 \#}$, Yuzhen Zheng ${ }^{2 \#}$, Yuming Rong ${ }^{3 \#}$, Xiaoqiang Li $^{4}$, Qihang Yan $^{1}$, Fangfang Duan ${ }^{3}$, \\ Zerui Zhao ${ }^{1}$, Jianlong Chen ${ }^{5}$, Shuqin Dai ${ }^{6}$, Junye Wang ${ }^{1}$
}

${ }^{1}$ Department of Thoracic Surgery, State Key Laboratory of Oncology in South China, Collaborative Innovation Center for Cancer Medicine, Sun Yat-sen University Cancer Center, Guangzhou, China; ${ }^{2}$ Department of Thoracic Surgery, Sun Yat-sen University Sixth Affiliated Hospital, Guangzhou, China; ${ }^{3}$ VIP Region, State Key Laboratory of Oncology in South China, Collaborative Innovation Center for Cancer Medicine, Sun Yat-sen University Cancer Center, Guangzhou, China; ${ }^{4}$ Department of Thoracic Surgery, Peking University Shenzhen Hospital, Shenzhen, China; ${ }^{5}$ Department of Thoracic Surgery, The Second People's Hospital of Shan Tou, Shantou, China; ${ }^{6}$ Department of Laboratory Medicine, State Key Laboratory of Oncology in South China, Collaborative Innovation Center for Cancer Medicine, Sun Yat-sen University Cancer Center, Guangzhou, China

Contributions: (I) Conception and design: J Wang, S Dai, W Zhai; (II) Administrative support: J Wang; (III) Provision of study materials or patients: Y Zheng, Y Rong, W Zhai; (IV) Collection and assembly of data: Y Zheng, Y Rong, X Li, Q Yan, F Duan, J Chen; (V) Data analysis and interpretation: Z Zhao, W Zhai; (VI) Manuscript writing: All authors; (VII) Final approval of manuscript: All authors.

"These authors contributed equally to this work.

Correspondence to: Junye Wang; Shuqin Dai. State Key Laboratory of Oncology in South China, Collaborative Innovation Center for Cancer Medicine, Sun Yat-sen University Cancer Center, 651 Dongfeng Road East, Guangzhou 510060, China.

Email: wangjy@sysucc.org.cn; daishq@sysucc.org.cn.

Background: The examination of lymph nodes (LNs) is critical for accurate node staging in patients with non-small cell lung cancer (NSCLC), but a consensus on the examinations of hilar and intrapulmonary (N1 station) LNs has not been reached. This study aimed to evaluate the role of LN dissection and pathological examination of N1 LN stations and their effects on survival in patients with stage IA-IIA NSCLC.

Methods: Data from patients pathologically staged as IA-IIA who underwent radical surgery and confirmed as lacking LN metastases from January 2008 to March 2018 were retrospectively reviewed. The KaplanMeier method was used to determine the overall survival (OS) and disease-free survival (DFS). After propensity score matching (PSM), a Cox model was used to determine the prognostic factors.

Results: Of the 1,935 patients investigated, the median number of N1 stations examined was 3. Patients with at least $2 \mathrm{~N} 1$ stations examined had apparently better OS $(\mathrm{P}=0.002)$ and DFS $(\mathrm{P}=0.001)$. All patients were divided into patients with $0-1 \mathrm{~N} 1$ station examined and patients with 2-5 N1 stations examined. After PSM, the number of N1 stations examined was an independent prognostic factor for DFS $(\mathrm{P}=0.004)$. Patients with 2-5 N1 stations examined experienced prolonged DFS ( $\mathrm{P}=0.010)$. Patients in group 12 experienced prolonged OS $(\mathrm{P}=0.021)$ and DFS $(\mathrm{P}=0.026)$. Patients in group 13 or 14 experienced prolonged $\mathrm{OS}(\mathrm{P}=0.028)$. Conclusions: A larger extent of N1 station examination was associated with prolonged DFS in patients with stage IA-IIA NSCLC after lobectomy. The dissection and examination of at least 2 N1 stations included LNs from the lobar and segmental drainage fields.

Keywords: Non-small cell lung cancer (NSCLC); lymph node (LN); N1; propensity-score matching; prognosis

Submitted Dec 30, 2020. Accepted for publication Feb 24, 2021.

doi: $10.21037 /$ jtd-20-3611

View this article at: http://dx.doi.org/10.21037/jtd-20-3611 


\section{Introduction}

Lung cancer is the leading cause of cancer-related death worldwide, and approximately $85 \%$ of patients are diagnosed with non-small cell lung cancer (NSCLC) $(1,2)$. Greater extensive application of low-dose computed tomography (LDCT) is associated with increasing rate early-stage NSCLC diagnosis, which is associated with a reduction in mortality from lung cancer $(3,4)$. For patients with early-stage NSCLC, the 5-year survival rate ranges from $60-92 \%(5,6)$.

An examination of lymph nodes (LNs) is critical for accurate node staging, which determines whether postoperative patients require adjuvant therapy (7). Lobectomy with systematic LN dissection or systematic LN sampling remains the standard therapy for early-stage resectable NSCLC (8). The National Comprehensive Cancer Network (NCCN) guidelines recommend the dissection or sampling of at least 3 stations of mediastinal LNs (N2) as a routine part of lung cancer surgeries (9). Recently, based on the theory of lobe-specific lymphatic drainage regions, studies analyzing survival have shown that selective mediastinal LN dissection for early NSCLC had a similar impact to systematic mediastinal LN dissection in terms of long-term survival (10-12). However, for intrapulmonary or hilar (N1) LNs, a consensus on the N1 stations examined has not been reached. For patients with early-stage disease, sublobar resection is widely accepted but is associated with a $39 \%$ increased risk of cancer recurrence, as reported by Subramanian et al. (13). They also mentioned that patients who underwent sublobar resection had an inadequate $\mathrm{LN}$ examination. Therefore, in this study, we aimed to evaluate the roles of surgical $\mathrm{LN}$ dissection and pathological examination of N1 LN stations and their effects on the overall survival (OS) and disease-free survival (DFS) of patients who underwent lobectomies and LN dissection for stage IA-IIA NSCLC. We present the following article in accordance with the STROBE reporting checklist (available at http://dx.doi.org/10.21037/jtd-20-3611).

\section{Methods}

\section{Patients}

The study was conducted in accordance with the Declaration of Helsinki (as revised in 2013). This study was approved by the Institutional Review Board of Sun Yat-sen University Cancer Center (IRB No. SZR2019-108). The requirement to obtain informed consent from the patient was waived. We retrospectively screened 2,911 patients with early-stage NSCLC who underwent radical resection in the thoracic surgery department of our institution from January 2008 to March 2018. The inclusion criteria were: (I) a confirmed pathological diagnosis of stage T1a-T2bN0M0 (IA-IIA) NSCLC; (II) preoperative clinical confirmation of a lack of LN metastasis; (III) at least one N2 station examined; and (IV) R0 resection. The following exclusion criteria were used: (I) patients who received any form of neoadjuvant therapy; (II) had multiple primary cancers, including lung tumors as the second primary cancer; (III) a DFS or OS time was less than one month; and (IV) patients who underwent any form of sublobectomy. All patients were restaged according to the TNM staging criteria of the $8^{\text {th }}$ NCCN staging system.

\section{Surgical approach, follow-up, and survival analysis}

In our center, the dissection of $\mathrm{N} 1$ and $\mathrm{N} 2$ station LNs was performed by the surgeons. Surgeons chose systematic $\mathrm{LN}$ dissection, LN sampling or selective mediastinal $\mathrm{LN}$ dissection according to the general condition of the patients, the stage of the tumor and their own habits. Hilar (group 10) LNs were also dissected by surgeons during the operation. Interlobar (group 11), lobar (group 12), segmental (group 13) and subsegmental (group 14) LNs were carefully dissected ex vivo from dissected lung specimens by surgeons, and each LN station was examined independently. Pathologists evaluated the number and quality of LNs and confirmed the results of the pathological examination. We reviewed the pathology reports of each patient to examine the number of LNs and LN stations. Some patients received adjuvant chemotherapy, and whether a patient received ACT was determined at the discretion of the attending doctor. All patients attended follow-up visits every 3 months in the first two years after surgery, every 6 months until 5 years and once a year thereafter. During each follow-up, patients had complete medical history reviews and physical and full laboratory check-ups. A chest and upper abdomen CT scan was also routinely prescribed. The study endpoints were OS and DFS, which were defined as the time from surgery to death and the time from surgery to first locoregional or distant recurrence or to cancerrelated death, respectively.

\section{Statistical analysis}

Statistical analyses to identify risk characteristics were 
A

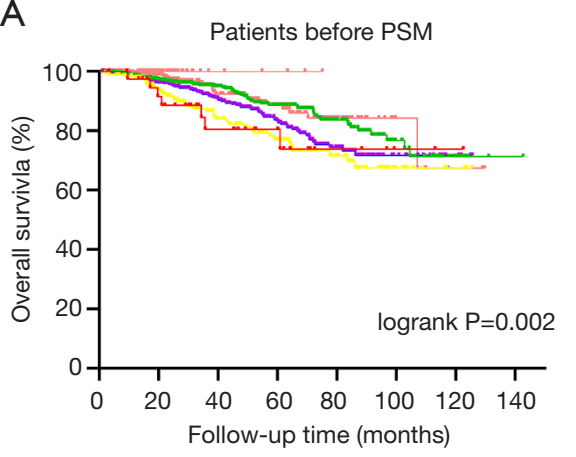

C

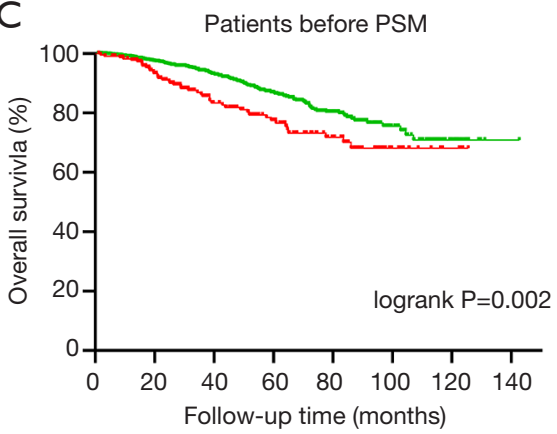

B
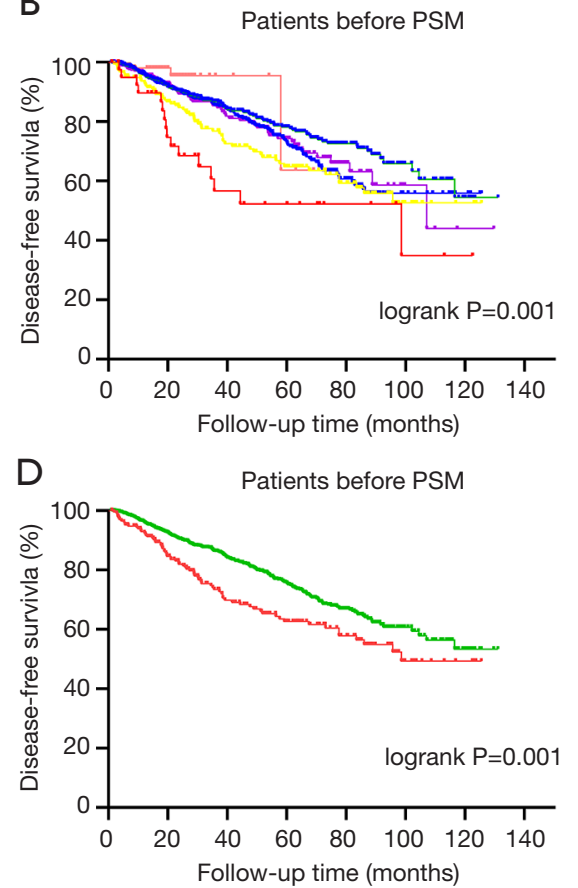

Follow-up time (months)

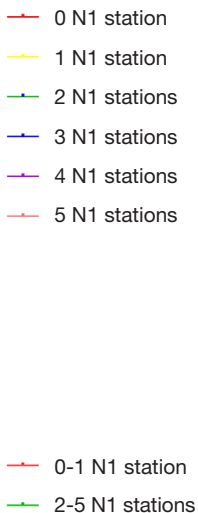

- 0-1 N1 station

- 2-5 N1 stations

Figure 1 Kaplan-Meier survival curves for patients with different number of N1 stations examined before PSM. (A) OS for patients with 0-5 N1 stations examined; (B) DFS for patients with 0-5 N1 stations examined; (C) OS for patients with 0-1 and 2-5 N1 stations examined; (D) DFS for patients with $0-1$ and 2-5 N1 stations examined before. PSM, propensity score matching; OS, overall survival; DFS, diseasefree survival.

performed using the SPSS 22.0 software package (SPSS, Inc., Chicago, IL). T-tests were performed to compare quantitative data, and Pearson's $\chi^{2}$ or Kruskal-Wallis $\mathrm{H}$ tests were used to compare categorical data between two groups. Next, 1:1 propensity score matching (PSM) was conducted to reduce the possible effects of selection bias, and the propensity score was calculated using a logistic regression model (caliper $=0.10$ ). The final covariates were age, sex, tumor size, $8^{\text {th }}$ TNM stage, visceral pleural invasion (including PL1 and PL2), histology, differentiation degree, lymphovascular invasion, surgical approach, number of N2 LNs examined, thoracotomy or video-assisted thoracoscopic surgery (VATS), and number of N2 stations examined. OS and DFS were calculated using the KaplanMeier method and compared with the log-rank test. A Cox proportional hazards regression model was constructed to determine prognostic factors. All factors with $\mathrm{P}<0.05$ in the univariate analysis were included in the multivariate analysis. Statistical significance was set to a $\mathrm{P}$ value $<0.05$, and all hypotheses were two-sided.

\section{Results}

\section{Patient characteristics}

At the beginning of this study, both the $\mathrm{OS}(\mathrm{P}=0.002)$ (Figure $1 A)$ and DFS $(\mathrm{P}=0.001)$ (Figure $1 B)$ of patients with different numbers of N1 stations examined were significantly different, and patients with at least $2 \mathrm{~N} 1$ stations examined experienced a prolonged OS $(\mathrm{P}=0.002)$ (Figure $1 C)$ and DFS $(\mathrm{P}=0.001)$ (Figure 1D). Therefore, we divided all patients into two groups: 233 patients (12\%) with $0-1 \mathrm{~N} 1$ station examined and 1,702 patients (88\%) with $2-5 \mathrm{~N} 1$ stations examined. A total of 1,935 patients were enrolled in this study, with a median age of 61 years (range from 20 to 83 years). The median follow-up time was 60 months. A total of 1,493 patients suffered from adenocarcinoma, 297 suffered from squamous cell carcinoma, and 145 patients with other histologies included 49 with adenosquamous carcinoma, 41 with lymphoepithelioma-like carcinoma, 5 with large cell carcinoma, 5 with carcinoid carcinoma, 6 with mixed neuroendocrine carcinoma, 6 with sarcomatoid 
carcinoma and 33 with other rare histological types or undifferentiated tumors. A total of 1,752 patients underwent lobectomy (90.5\%), 118 patients underwent lobectomy combined with sublobectomy in other lobes (6.1\%), 35 patients underwent lobectomy in two different lobes (1.8\%), and 30 patients underwent pneumectomy (1.6\%). More than half of the patients $(n=1,054)$ underwent VATS, and 880 patients underwent thoracotomy. Three hundred fortytwo patients received adjuvant chemotherapy. The median number of $\mathrm{N} 2$ stations examined was 3 , and the median number of N2 LNs examined was 11. The mean number of $\mathrm{N} 2$ stations examined was 3.3 (ranging from 1 to 8), and the mean number of $\mathrm{N} 2 \mathrm{LNs}$ examined was 13.2 (ranging from 1 to 104). The numbers of patients with one, two, three, four and five N1 stations examined were 192 (9.9\%), 512 (26.5\%), 664 (34.3\%), 420 (21.7\%) and 106 (5.5\%), respectively. Patients with $0-1 \mathrm{~N} 1$ station examined were older $(\mathrm{P}=0.014)$ and had a smaller number of $\mathrm{N} 2$ stations $(\mathrm{P}=0.024)$ and $\mathrm{N} 2 \mathrm{LNs}(\mathrm{P}=0.004)$ examined. After PSM, 232 pairs of patients were matched at a 1:1 ratio. Table 1 shows the characteristics of the investigated patients.

\section{Survival analysis}

After PSM, a significant difference in OS was not observed among patients with different numbers of $\mathrm{N} 1$ stations examined $(\mathrm{P}=0.505)$ (Figure $2 A$ ), and the examination of 2-5 $\mathrm{N} 1$ stations did not noticeably increase $\mathrm{OS}(\mathrm{P}=0.070)$ (Figure $2 B)$. However, the difference in the DFS of patients with different numbers of $\mathrm{N} 1$ stations examined was significant $(\mathrm{P}=0.041$ ) (Figure $2 C$ ), and patients with at least $2 \mathrm{~N} 1$ stations examined experienced a prolonged DFS than patients with $0-1 \mathrm{~N} 1$ station examined $(\mathrm{P}=0.010)$ (Figure $2 D)$. We used a Cox proportional hazards model to determine the independent prognostic factors for DFS. The univariate and multivariate analyses of OS are shown in the Table S1. All factors except histology, the number of N2 LNs examined and the number of $\mathrm{N} 2$ stations examined were included in the multivariate analysis. We identified sex $(\mathrm{HR}=0.578$, 95\% CI, 0.360-0.928, $\mathrm{P}=0.023$ ), age ( $\mathrm{HR}=1.026,95 \% \mathrm{CI}$, $1.004-1.048, \mathrm{P}=0.017), 8^{\text {th }}$ TNM stage $(\mathrm{HR}=1.703,95 \% \mathrm{CI}$, 1.362-2.129, $\mathrm{P}<0.001)$ and number of $\mathrm{N} 1$ stations examined ( $2 \mathrm{~N} 1$ vs. 0 N1: HR $=0.278,95 \%$ CI, $0.135-0.574, \mathrm{P}=0.001$; $3 \mathrm{~N} 1$ vs. $0 \mathrm{~N} 1$ : $\mathrm{HR}=0.846,95 \%$ CI, 0.775-0.923, $\mathrm{P}<0.001$ ) as independent prognostic factors (Table 2).

We further analyzed the effect of the examination of a single N1 station on survival to determine which lymphatic drainage field should receive special attention in the N1 LN examination. Because group 13 and 14 LNs are sometimes indistinguishable, we combined them into one group. Patients with an examination of group $12 \mathrm{LNs}$ experienced significantly prolonged $\mathrm{OS}(\mathrm{P}=0.021)$ (Figure $3 A)$ and $\mathrm{DFS}$ $(\mathrm{P}=0.026)$ (Figure $3 B)$ than patients in whom group $12 \mathrm{LNs}$ were not examined. Patients with an examination of LNs in group 13 or 14 experienced significantly prolonged OS $(\mathrm{P}=0.028)$ (Figure 3C) but not DFS $(\mathrm{P}=0.214)$ (Figure $3 D$ ) than patients without an examination of group 13 LNs. OS and DFS were not apparently different in patients with or without examinations of LNs from group 10, group 11 and group 14 (Figure S1).

\section{Discussion}

For patients with early-stage NSCLC, surgical resection is the preferred therapy, and the examination of LNs is critical for accurate staging. For patients who are LN negative, the scope of the LN examination remains controversial. The NCCN guidelines recommend an appropriate examination of the N1 LN but do not tell us what is appropriate. In this study, we found that the number of N1 stations examined was an independent prognostic factor for DFS in patients with T1-2N0 disease. Patients with at least two N1 stations examined experienced a significantly longer DFS than patients with $0-1 \mathrm{~N} 1$ station examined. The results of this study are potentially a useful complement to the guidelines.

Previous studies have mentioned that an adequate LN examination can increase the accuracy of $\mathrm{N}$ staging and improve long-term survival (14-17). Ou et al. reported better outcomes for patients with stage IA NSCLC in whom more LNs were examined. They suggested the removal of at least 15 LNs for optimal survival prognostication. However, $\mathrm{N} 1$ and N2 LN stations were not independently analyzed in their study (14). Doddoli et al. reported that two N2 stations sampled with at least 10 assessed LNs improved the prediction of OS and did not increase short-term surgical mortality (15). Dai et al. also confirmed that the retrieval of $>10$ LNs was a favorable prognostic factor for OS (17) but failed to evaluate the impact of the $\mathrm{N} 1$ station.

A retrospective study of 119 patients with T1N0 disease found that the local recurrence-free survival of patients with T1N0 disease and an inadequate examination N1 stations was similar to patients with $\mathrm{T} 1 \mathrm{~N} 1$ disease (18). Consistent with the findings of our study, the aforementioned study showed that patients from whom one N1 station was removed experienced prolonged DFS compared with patients with no N1 stations evaluated but 
Table 1 Patients characteristics

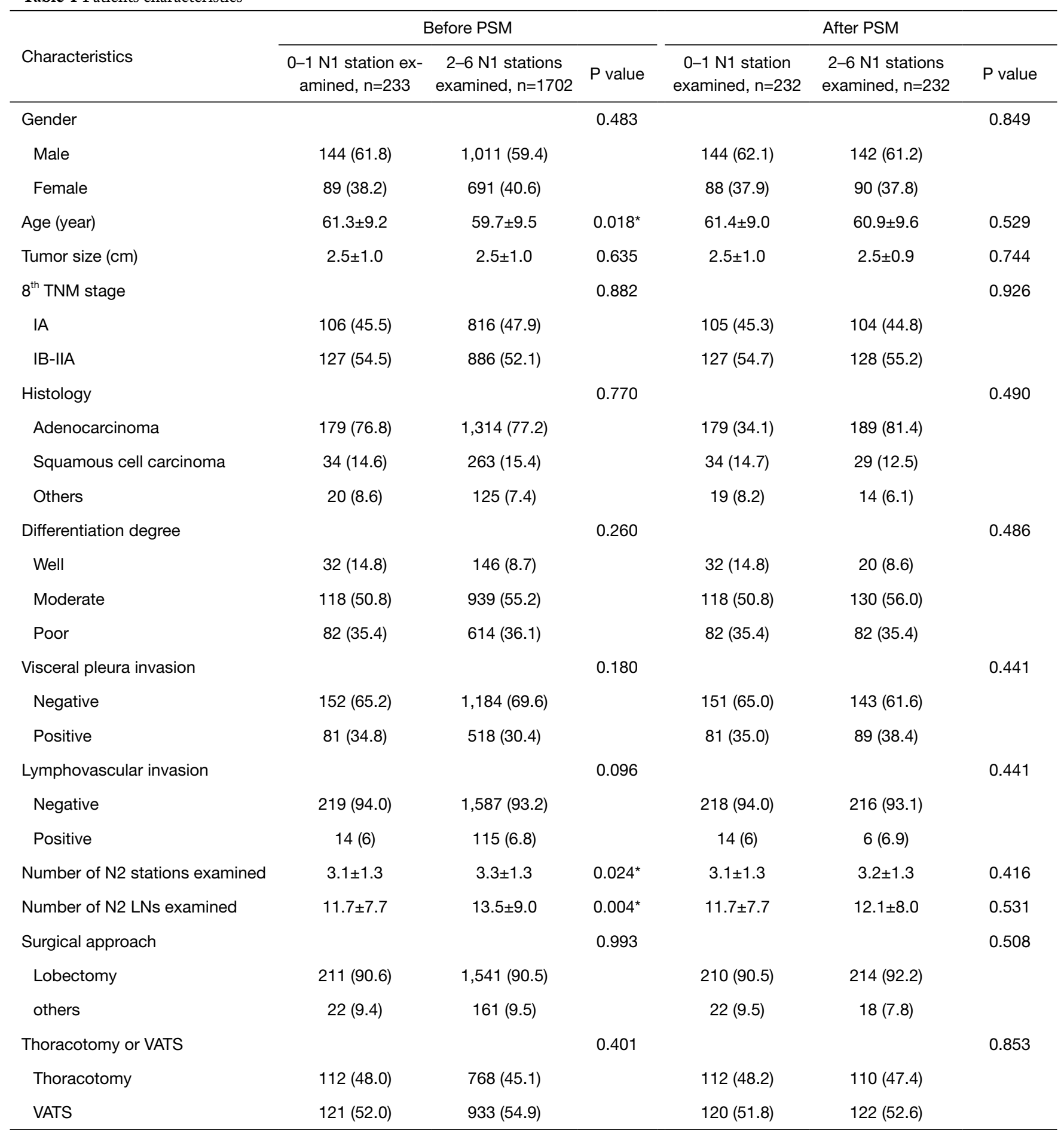

*, P<0.05. PSM, propensity score matching; VATS, video-assisted thoracoscopic surgery. 

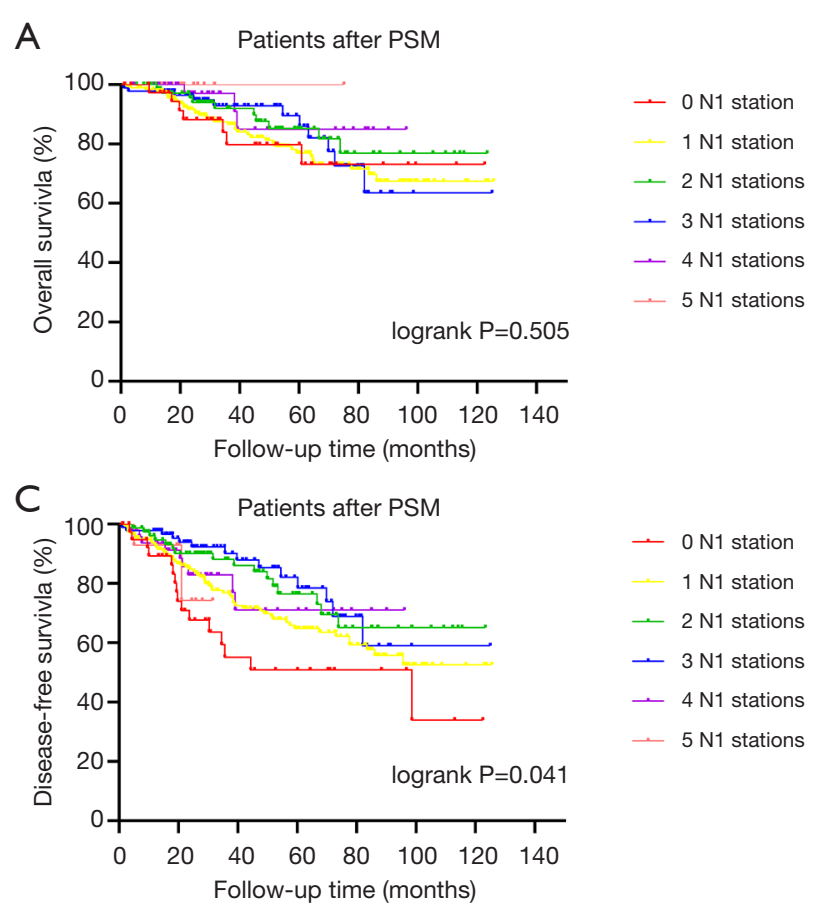
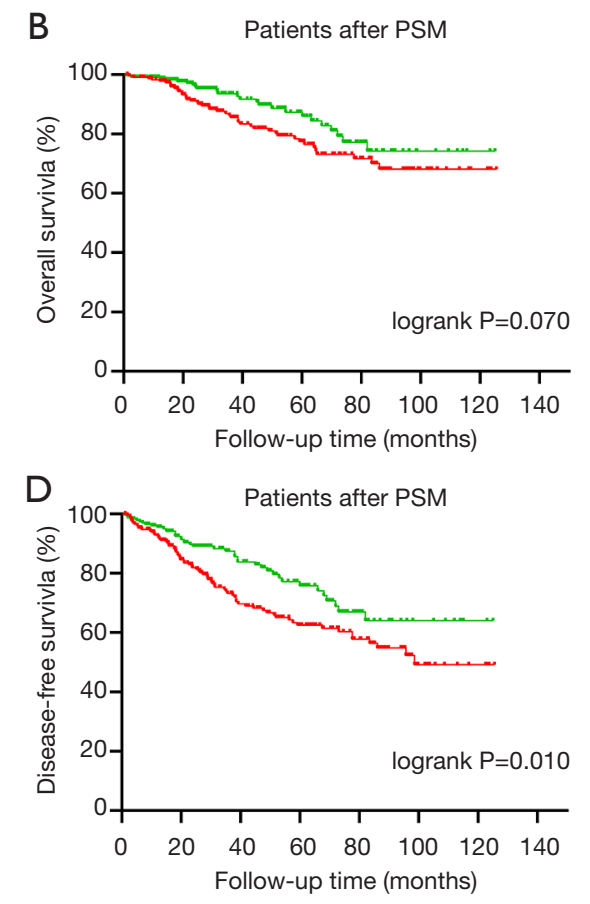

- 0-1 N1 station

- 2-5 N1 stations

Figure 2 Kaplan-Meier survival curves for patients with different number of N1 stations examined after PSM. (A) OS for patients with 0-5 N1 stations examined; (B) OS for patients with 0-1 and 2-5 N1 stations examined; (C) DFS for patients with 0-5 N1 stations examined; (D) DFS for patients with $0-1$ and 2-5 N1 stations examined. PSM, propensity score matching; OS, overall survival; DFS, disease-free survival.

still did not answer the question about the extent of $\mathrm{N} 1$ station dissection. A study from the American College of Surgeons Oncology Group (ACOSOG) Z0030 (Alliance) trial also reported a trend toward a survival advantage by examining a greater number of intrapulmonary LNs (19). These 2 studies indicated that the examination of the N1 station was associated with an improved long-term prognosis. In addition, several studies mentioned a phenomenon in which the extent and quality of both N2 and N1 LN evaluations displayed remarkable discrepancies during the surgical treatment of lung cancer $(18,20,21)$. A hypothesis-confirming experiment revealed that $60 \%$ of intrapulmonary LNs were routinely discarded without examination, including up to $12 \%$ of $\mathrm{pN} 0$ specimens (22). These phenomena reflect the lack of consensus on the examination of $\mathrm{N} 1$ stations.

In this study, we first confirmed that the number of N1 stations examined was positively associated with DFS. Further survival analyses showed that patients with examinations of group 12, 13 and 14 LNs had survival advantages. Some researchers have explained that patients who have more LNs examined achieve better outcomes. The examination of a greater number of $\mathrm{N} 1$ stations represents a large extent of N1 LNs examined, which might result in more accurate staging of earlystage NSCLC (7). The stage migration effect is also a reasonable explanation for these results in the present study. An inadequate examination of N1 stations may result in the misclassification of some patients with a true N1 stage as patients with N0 stage disease, leading to undertreatment. Patients who should have received adjuvant therapy would not receive it, thereby leading to a poor survival outcome. These patients with shorter survival who should have been classified as N1 stage resulted in an incorrect estimation of the survival time. Some previous studies also identified the potential of resecting unidentified micrometastases as another mechanism explaining these results $(21,23)$. However, in this study, no patients underwent sublobectomy. Micrometastases in intrapulmonary LNs were removed along with the lobe. The resection of unidentified micrometastases might not be a proper interpretation of the results from this study. Wang et al. reviewed 442 patients with pN0 disease who underwent radical resection and found that patients with examinations of LNs in groups 10-14 groups experienced longer $\mathrm{OS}(\mathrm{P}=0.027)$ and DFS $(\mathrm{P}=0.021)$ than patients 
Table 2 Univariate and multivariate analysis of DFS for patients after PSM

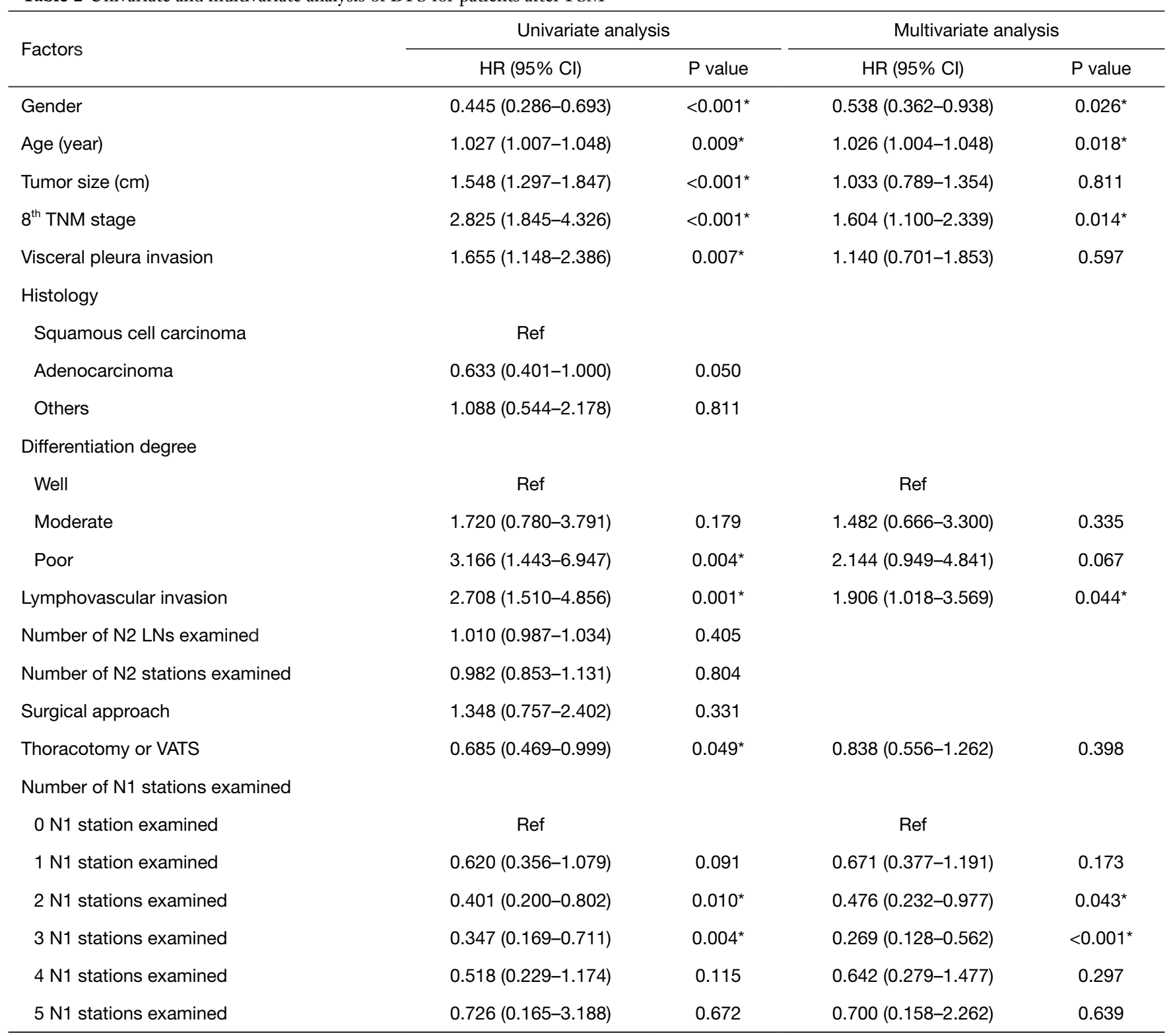

*, P<0.05. DFS, disease-free survival; PSM, propensity score matching; VATS, video-assisted thoracoscopic surgery.

with examinations of LNs in groups 10-12 only (24). This finding suggests the importance of examining LNs from the drainage area of the bronchi segments for the accurate staging of patients with early-stage NSCLC.

The reliability of the data and analyses reported in the present study are that the dissection of N1 LNs was performed by surgeons, and pathologists performed the pathological examination. This process of LN examination can enhance the detection rate of intrapulmonary LNs. A previous study in the community-based Memphis
Metropolitan Area Quality of Surgical Resection cohort related to an incomplete intrapulmonary LN examination that solely relied on pathologists for the examination showed that a mediocre median of $4 \mathrm{~N} 1 \mathrm{LNs}$ were removed for the pathological examination (25). The median number of N1 LNs examined was 5 in another cohort of the ACOSOG Z0030 trial (26). In the present study, the median number of examined N1 LNs was 8. To some extent, a large number of N1 LNs examined was associated with a larger number of N1 stations examined, showing that patients in 

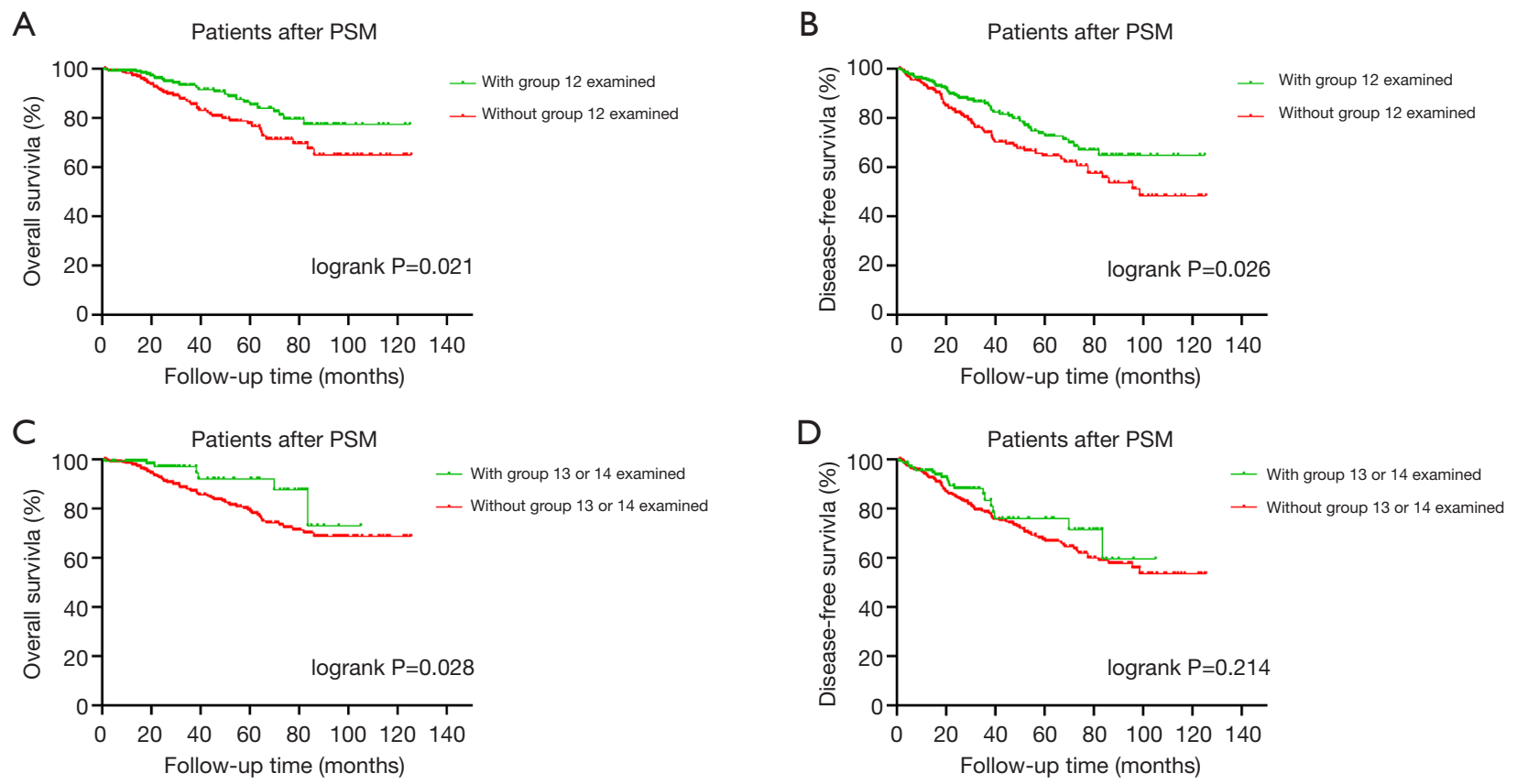

Figure 3 Kaplan-Meier survival curves for patients with a single N1 station examined after PSM. (A) OS for patients with and without group 12 examined; (B) DFS for patients with and without group 12 examined; (C) OS for patients with and without group 13 or 14 examined; (D) DFS for patients with and without group 13 or 14 examined. PSM, propensity score matching; OS, overall survival; DFS, disease-free survival.

this study may have had more accurate $\mathrm{N}$ staging.

However, this study documented the effect of the number of N1 LNs examined on the survival of patients who underwent lobectomy. For patients who received sublobar resection, the effect of the number of N1 LNs examined is still being debated. Segmentectomy is an anatomical sublobar resection technique that is superior in dissecting intrapulmonary LNs, especially in dissecting group 12 and group $13 \mathrm{LNs}$, which can provide a relatively large extent of the examined N1 stations. However, wedge resection has limitations in the number of N1 LNs examined because wedge resection is a nonanatomical resection technique. Altorki et al. reviewed 160 patients with cT1N0M0 disease who underwent wedge resection and 129 patients who underwent segmentectomy and found that more N1 stations were examined in patients who underwent segmentectomy $(\mathrm{P}<0.001)$ (27). Ding et al. reviewed 2,864 patients with primary tumors $<2 \mathrm{~cm}$ from the SEER database, including 2,298 patients who underwent wedge resection and 566 patients who underwent segmentectomy, and discovered that segmentectomy was significantly associated with the removal of more LNs (28). He et al. found that at least 3 LNs must be examined in patients undergoing sublobar resection by analyzing 16,630 lobectomies, 3,979 wedge resections, and 1,119 segmentectomies from the SEER database. They also proposed that a greater number of LNs examined potentially explained why segmental resection resulted in a survival advantage compared with wedge resection (29). However, Sienel et al. revealed that the surgical approach (segmentectomy versus wedge resection) instead of the number of LNs examined was an independent prognostic factor for stage IA NSCLC (30). More research is needed to determine the extent of LNs examined in patients undergoing sublobectomy.

The major limitation of this single-center retrospective study is that patient selection bias inevitably exists. Patients with 0-1 N1 station examined included more elderly patients, which may affect the results of survival analyses. Therefore, we used PSM and multivariate analyses to eliminate bias as much as possible, but further validation from a multicenter database is needed. In addition, the data from this study did not identify a positive effect of the number of $\mathrm{N} 2$ stations examined on the prognosis, and we were unable to determine the optimal number of $\mathrm{N} 2$ stations that should be examined. Furthermore, the pattern of recurrence and how it was affected by the number of N1 
stations examined are valuable topics to be investigated. However, the pattern of recurrence was unavailable in some patients, and we were unable to analyze how recurrence was affected by the number of $\mathrm{N} 1$ stations examined.

\section{Conclusions}

For patients with stage I-IIA NSCLC who underwent lobectomy, a larger extent of N1 station examination was associated with prolonged DFS. Based on our findings, we recommend the dissection and pathological examination of at least $2 \mathrm{~N} 1$ stations. In particular, the LNs from the lobar and segmental drainage fields should be dissected and pathologically examined. These findings should be confirmed in a large, prospective randomized clinical study.

\section{Acknowledgments}

Funding: This work was supported by the Natural Science Foundation of Guangdong Province of China (Grant Numbers. 2019A1515011601, 2019A1515010298) the $\mathrm{Wu}$ Jieping Medical Foundation (320.6750.2020-15-4).

\section{Footnote}

Reporting Checklist: The authors have completed the STROBE reporting checklist. Available at http://dx.doi. org/10.21037/jtd-20-3611

Data Sharing Statement: Available at http://dx.doi. org/10.21037/jtd-20-3611

Peer Review File: Available at http://dx.doi.org/10.21037/jtd20-3611

Conflicts of Interest: All authors have completed the ICMJE uniform disclosure form (available at http://dx.doi. org/10.21037/jtd-20-3611). The authors have no conflicts of interest to declare.

Ethical Statement: The authors are accountable for all aspects of the work in ensuring that questions related to the accuracy or integrity of any part of the work are appropriately investigated and resolved. The study was conducted in accordance with the Declaration of Helsinki (as revised in 2013). This study was approved by the Institutional Review Board of Sun Yat-sen University Cancer Center (IRB No. SZR2019-108). The requirement to obtain informed consent from the patient was waived.

Open Access Statement: This is an Open Access article distributed in accordance with the Creative Commons Attribution-NonCommercial-NoDerivs 4.0 International License (CC BY-NC-ND 4.0), which permits the noncommercial replication and distribution of the article with the strict proviso that no changes or edits are made and the original work is properly cited (including links to both the formal publication through the relevant DOI and the license). See: https://creativecommons.org/licenses/by-nc-nd/4.0/.

\section{References}

1. Torre LA, Bray F, Siegel RL, et al. Global cancer statistics, 2012. CA Cancer J Clin 2015;65:87-108.

2. Herbst RS, Heymach JV, Lippman SM. Lung cancer. N Engl J Med 2008;359:1367-80.

3. Gou HF, Liu Y, Yang TX, et al. Necessity of organized low-dose computed tomography screening for lung cancer: From epidemiologic comparisons between China and the Western nations. Oncotarget 2017;8:1788-95.

4. Aberle DR, Adams AM, Berg CD, et al. Reduced lungcancer mortality with low dose computed tomographic screening. N Engl J Med 2011;365:395-409.

5. Goldstraw P, Chansky K, Crowley J, et al. The IASLC Lung Cancer Staging Project: Proposals for Revision of the TNM Stage Groupings in the Forthcoming (Eighth) Edition of the TNM Classification for Lung Cancer. J Thorac Oncol 2016;11:39-51.

6. Wang Z, Wang Y, Huang Y, et al. Challenges and research opportunities for lung cancer screening in China. Cancer Commun (Lond) 2018;38:34.

7. Massard G, Ducrocq X, Kochetkova EA, et al. Sampling or node dissection for intraoperative staging of lung cancer: a multicentric cross-sectional study. Eur J Cardiothorac Surg 2006;30:164-7.

8. Howington JA, Blum MG, Chang AC, et al. Treatment of stage I and II non-small cell lung cancer: Diagnosis and management of lung cancer, 3 rd ed: American College of Chest Physicians evidence-based clinical practice guidelines. Chest 2013;143:e278S-313S.

9. National Comprehensive Cancer Network. NCCN clinical practice guidelines in oncology. Non-small cell lung cancer (version 1, 2020). Available online: https:// www.nccn.org/ professionals/physician_gls/pdf/nscl.pdf

10. Ishiguro F, Matsuo K, Fukui T, et al. Effect of selective lymph node dissection based on patterns of lobe-specific 
lymph node metastases on patient outcome in patients with resectable non-small cell lung cancer: a large-scale retrospective cohort study applying a propensity score. J Thorac Cardiovasc Surg 2010;139:1001-6.

11. Jiang W, Chen X, Xi J, et al. Selective mediastinal lymphadenectomy without intraoperative frozen section examinations for clinical stage I non-small-cell lung cancer: retrospective study of 403 cases. World J Surg 2013;37:392-7.

12. Zhang Z, Miao J, Chen Q, et al. Assessment of non-lobespecific lymph node metastasis in clinical stage IA nonsmall cell lung cancer. Thorac Cancer 2019;10:1597-604.

13. Subramanian M, McMurry T, Meyers BF, et al. LongTerm Results for Clinical Stage IA Lung Cancer: Comparing Lobectomy and Sublobar Resection. Ann Thorac Surg 2018;106:375-81.

14. Ou SH, Zell JA. Prognostic significance of the number of lymph nodes removed at lobectomy in stage IA non-small cell lung cancer. J Thorac Oncol 2008;3:880-6.

15. Doddoli C, Aragon A, Barlesi F, et al. Does the extent of lymph node dissection influence outcome in patients with stage I non-small-cell lung cancer? Eur J Cardiothorac Surg 2005;27:680-5.

16. Jin K, Zhang K, Zhou F, et al. Selection of candidates for surgery as local therapy among early-stage small cell lung cancer patients: a population-based analysis. Cancer Commun (Lond) 2018;38:5.

17. Dai Y, Su XD, Long H, et al. Survival analysis of 220 patients with completely resected stage-II non-small cell lung cancer. Chin J Cancer 2010;29:538-44.

18. Saynak M, Hubbs J, Nam J, et al. Variability in defining T1N0 non-small cell lung cancer impacts locoregional failure and survival. Ann Thorac Surg 2010;90:1645-9; discussion 1649-50.

19. Osarogiagbon RU, Decker PA, Ballman K, et al. Survival Implications of Variation in the Thoroughness of Pathologic Lymph Node Examination in American College of Surgeons Oncology Group Z0030 (Alliance). Ann Thorac Surg 2016;102:363-9.

20. Farjah F, Flum DR, Thomas K, et al. Varghese. Surgeon specialty and long-term survival after pulmonary resection for lung cancer. Ann Thorac Surg 2009;87:995-1004; discussion 995-6.

21. Samayoa AX, Pezzi TA, Pezzi CM, et al. Rationale for a Minimum Number of Lymph Nodes Removed with Non-Small Cell Lung Cancer Resection: Correlating the Number of Nodes Removed with Survival in 98,970 Patients. Ann Surg Oncol 2016;23:1005-11.

22. Smeltzer MP, Faris N, Yu X, et al. Missed Intrapulmonary
Lymph Node Metastasis and Survival After Resection of Non-Small Cell Lung Cancer. Ann Thorac Surg 2016;102:448-53.

23. May M, Herrmann E, Bolenz C, et al. Association Between the Number of Dissected Lymph Nodes During Pelvic Lymphadenectomy and Cancer-Specific Survival in Patients with Lymph Node-Negative Urothelial Carcinoma of the Bladder Undergoing Radical Cystectomy. Ann Surg Oncol 2011;18:2018-25.

24. Wang X, Yan S, Lv C, et al. Impact of Omission of Intrapulmonary Lymph Node Retrieval on Outcome Evaluation of Lung Cancer Patients Without Lymph Node Metastasis: A Propensity Score Matching Analysis. Clin Lung Cancer 2017;18:e411-6.

25. Ramirez RA, Wang CG, Miller LE, et al. Incomplete intrapulmonary lymph node retrieval after routine pathologic examination of resected lung cancer. J Clin Oncol 2012;30:2823-8.

26. Darling GE, Allen MS, Decker PA, et al. Number of lymph nodes harvested from a mediastinal lymphadenectomy: results of the randomized, prospective American College of Surgeons Oncology Group Z0030 trial. Chest 2011;139:1124-9.

27. Altorki NK, Kamel MK, Narula N, et al. Anatomical Segmentectomy and Wedge Resections Are Associated with Comparable Outcomes for Patients with Small cT1N0 Non-Small Cell Lung Cancer. J Thorac Oncol 2016;11:1984-92.

28. Ding H, Wang H, Xu L, et al. Survival and Resected Lymph Node Number During Sublobar Resection for N0 Non-Small Cell Lung Cancer $2 \mathrm{~cm}$ or Less. Ann Thorac Surg 2019;107:1647-55.

29. He Z, Li Z, Xu S, et al. Prognostic Significance of Lymph Node Count Removed at Sublobar Resection in Pathologic Stage IA Non-Small-cell Lung Cancer: A PopulationBased Analysis. Clin Lung Cancer 2020. [Epub ahead of print]. doi: 10.1016/j.cllc.2020.09.015.

30. Sienel W, Dango S, Kirschbaum A, et al. Sublobar resections in stage IA non-small cell lung cancer: segmentectomies result in significantly better cancer-related survival than wedge resections. Eur J Cardiothorac Surg 2008;33:728-34.

Cite this article as: Zhai W, Zheng Y, Rong Y, Li X, Yan Q, Duan F, Zhao Z, Chen J, Dai S, Wang J. The impact of an N1 lymph node examination in patients with early-stage non-small cell lung cancer: a retrospective cohort study. J Thorac Dis 2021;13(4):2184-2193. doi: 10.21037/jtd-20-3611 

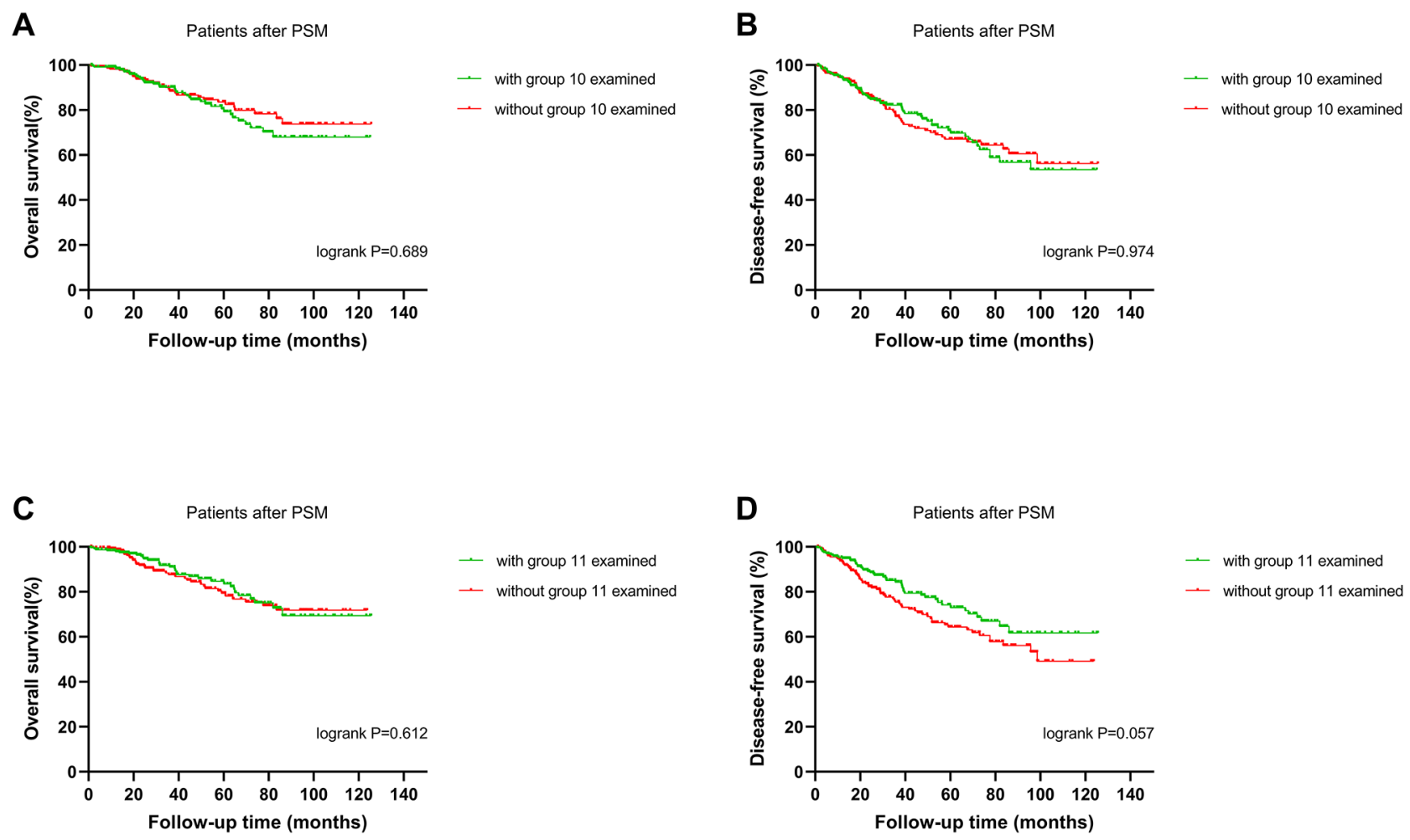

Figure S1 Kaplan-Meier survival curves for patients with a single N1 station examined after PSM. (A) OS for patients with and without group 10 examined; (B) DFS for patients with and without group 10 examined; (C) OS for patients with and without group 11 examined; (D) DFS for patients with and without group 11 examined. PSM, propensity score matching; OS, overall survival; DFS, disease-free survival. 
Table S1 Univariate and multivariate analysis of OS for patients after PSM

\begin{tabular}{|c|c|c|c|c|}
\hline Factors & \multicolumn{2}{|c|}{ Univariate analysis } & \multicolumn{2}{|c|}{ Multivariate analysis } \\
\hline Gender & $0.283(0.145-0.554)$ & $<0.001^{\star}$ & $0.349(0.172-0.706)$ & $0.003^{*}$ \\
\hline Age (year) & $1.037(1.010-1.065)$ & $0.007^{*}$ & $1.029(1.001-1.057)$ & $0.041^{*}$ \\
\hline Tumor size (cm) & $1.619(1.290-2.032)$ & $<0.001^{*}$ & $1.022(0.701-1.489)$ & 0.911 \\
\hline Visceral pleura invasion & $1.793(1.113-2.888)$ & $0.016^{\star}$ & $1.069(0.577-1.983)$ & 0.832 \\
\hline Histology & $0.937(0.850-1.034)$ & 0.196 & & \\
\hline Squamous cell carcinoma & Ref & & & \\
\hline Adenocarcinoma & $0.500(0.285-0.877)$ & $0.016^{\star}$ & $0.889(0.465-1.698)$ & 0.721 \\
\hline Well & Ref & & & \\
\hline Moderate & $1.106(0.458-2.671)$ & 0.823 & & \\
\hline Poor & $2.150(0.902-5.125)$ & $0.084^{*}$ & & \\
\hline Vascular invasion & $1.334(0.482-3.696)$ & 0.579 & & \\
\hline Number of N2 LNs examined & $1.005(0.973-1.037)$ & 0.777 & & \\
\hline Number of N2 stations examined & $0.934(0.776-1.124)$ & 0.468 & & \\
\hline Surgical approach & $1.850(0.945-3.620)$ & 0.073 & & \\
\hline Thoracotomy or VATS & $0.601(0.363-0.996)$ & $0.048^{\star}$ & $0.828(0.478-1.436)$ & 0.503 \\
\hline 3 N1 stations examined & $0.729(0.282-1.880)$ & 0.513 & & \\
\hline $4 \mathrm{~N} 1$ stations examined & $0.442(0.114-1.712)$ & 0.237 & & \\
\hline $5 \mathrm{~N} 1$ stations examined & $<0.001(<0.001)$ & 0.966 & & \\
\hline
\end{tabular}

*, P<0.05. OS, overall survival; PSM, propensity score matching; VATS, video-assisted thoracoscopic surgery. 